\title{
The Bird Community in a Coastal Wetland in East China and Its Spatial Responses to a Wind Farm
}

Yinrui Cheng ( $D$ cyrccc1226@163.com)

Nanjing Normal University School of Geography https://orcid.org/0000-0001-8754-3304

Yong Zha

Nanjing Normal University School of Geography

Wenmin Zhang

Nanjing Normal University School of Geography

Geng Wei

Nanjing Normal University School of Geography

Chuan Tong

Fujian Normal University

Dandan Du

Anhui Normal University

\section{Research Article}

Keywords: Dominance, Aquatic birds, Endangered species, Wind turbine, Coastal wetland

Posted Date: June 12th, 2021

DOI: https://doi.org/10.21203/rs.3.rs-573013/v1

License: (c) (i) This work is licensed under a Creative Commons Attribution 4.0 International License. Read Full License

Version of Record: A version of this preprint was published at Community Ecology on November 15th, 2021. See the published version at https://doi.org/10.1007/s42974-021-00065-4. 


\section{Abstract}

Coastal wetlands in East China are essential stopover places for birds along the East Asian-Australian Flyway. However, numerous wind turbines have been built in or near these wetlands in recent years, which might disturb the bird community in the area. Therefore, investigating the bird community and its responses to wind farms in coastal wetlands of East China is of great significance for bird conservation. In the spring and autumn of 2019 and 2020, we investigated the bird community in the Rudong coastal wetland in East China using point counts. We determined 4 geographical factors at each census point, i.e., distance to the wind farm boundary (DW), distance to the suburbs, distance to the sea, and vegetation area, and analysed the relationship between bird number and DW through partial correlation analysis. A total of 11 orders and 103 species of birds, including 4 endangered species, were observed during our survey. Charadriiformes was the dominant taxon in the wetland, and Calidris alpina was the most common species in both spring and autumn.

Passeriformes exhibited high species richness but low numbers. The results of partial correlation analysis indicated that birds' responses to the wind farm varied depending on their dominance and category: dominant and subdominant birds tended to avoid the wind farm, whereas rare birds tended to approach them; aquatic birds were alert to the wind farm, whereas terrestrial birds better adapted to them. We concluded that the dominant aquatic birds, including the endangered species Calidris tenuirostris, were most negatively impacted by the wind farm; the occasional birds and rare aquatic birds might be disturbed by wind farm but not significantly so; and the rare terrestrial birds were least disturbed by or even benefited from the wind farm.

\section{Introduction}

The coastal wetlands in East China are essential stopover places for birds along the East Asian-Australian Flyway (EAAF) (Cao et al., 2009; Yong et al., 2018). Numerous aquatic birds stop in these wetlands to accumulate energy reserves before or after they fly over the Yellow Sea (Fig. 1a) (Ma et al., 2006); moreover, some terrestrial birds also rest in these wetlands during their migration (Yong et al., 2015). For threatened species in the flyway, such as Calidris pygmaea and Tringa guttifer, these wetlands are crucial for future survival (Peng et al., 2017; Yang et al., 2020). In recent decades, bird surveys have been conducted in some coastal wetlands of East China (Ma et al., 2009; Bai et al., 2015; Peng et al., 2017). However, most of these surveys focused on shorebirds or endangered species, whereas investigations of entire communities are limited. Moreover, in recent years, birds in the area have been threatened by wetland loss caused by agricultural and industrial development (Lin and Yu, 2018; Jackson et al., 2021). Thus, further observations of the bird community are necessary for bird conservation in the area.

Wind energy is increasingly used for electricity generation because it is clean and renewable (Herbert et al., 2014; Kumar et al., 2016). Recent reports have shown that the capacity of the world's wind farms is growing at 10\% per year and reached $744 \mathrm{GW}$ by the end of 2020, covering more than $7 \%$ of the global electricity demand (WWEA, 2021). Despite the benefits of wind power generation, considerable research has shown that wind turbines are disadvantageous to birds. First, birds can be killed by collisions with wind turbines (Newton and Little, 2009; Grodsky et al., 2013). Bird carcass collection in a coastal wind farm showed that collisions accounted for 3\% of bird deaths (Newton and Little, 2009). Second, birds tend to avoid wind farms behaviourally (Plonczkier and Simms, 2012; Villegas-Patraca et al., 2014). Specifically, they usually change their migration routes and stopover sites due to wind farms, and this expenditure of energy could induce an increase in fatal casualties during their migration (Hilgerloh et al., 2011). Third, constructions of wind turbines and attached facilities can induce fragmentation and functional loss of bird habitat (Pruett et al., 2009; Marques et al., 2020), which is essential for bird foraging and breeding.

China is the largest energy consumer worldwide (Yang et al., 2017). In terms of sustainable development, China hosts over one-third of the world's wind power capacity (Yang et al., 2017; WWEA, 2021). In recent years, numerous wind farms have been established in the coastal areas of East China (He et al., 2016; Wang et al., 2019), which might disturb wetland bird communities. In this context, we wish to know the bird community composition in wetlands with wind farms in the region; 
we also wonder if all the species in the community tend to avoid the wind farms, and if not, whether we can uncover some rules underlying their responses. These issues have great significance for protecting birds in the EAAF. In this paper, we investigated the bird community in the Rudong coastal wetland of East China, where wind turbines have been installed, and analysed the relation between bird number and distance to wind farms. The main objectives of this study were to (1) provide community data for bird studies on the EAAF; (2) explore the implications of wind farms for bird communities; and (3) provide recommendations for future bird conservation in the area.

\section{Materials And Methods The study area}

Our research was conducted in the Rudong coastal wetland (120 $\left.56^{\prime} 32^{\prime \prime} \sim 121^{\circ} 12^{\prime} 35^{\prime \prime} \mathrm{E}, 32^{\circ} 29^{\prime} 47^{\prime \prime} \sim 32^{\circ} 39^{\prime} 12^{\prime \prime} \mathrm{N}\right)$, East China (Fig. 1a). The wetland has a northern subtropical monsoon climate, with an annual mean temperature of $14.8^{\circ} \mathrm{C}$ and an annual mean precipitation of $1029 \mathrm{~mm}$ (Li et al., 2018). The vegetated marshes are dominated by Spartina alterniflora. Tides in the region are semidiurnal. The wetland image shown in Fig. 1b was extracted from Landsat 8 OLI images (02:30:14Z, May 3rd, 2020), which were taken at the middle-tide level.

Many birds in the EAAF, including the endangered species Calidris pygmaea (critically endangered), Calidris tenuirostris, and Platalea minor, stop in the Rudong coastal wetland during their migration (Ma and Chen, 2018; Yang et al., 2020). Thus, the wetland is well-known to birdwatchers worldwide. However, hundreds of wind turbines (capacity of each turbine $\sim 3 \mathrm{MW}$, hub height $\sim 90 \mathrm{~m}$, rotor diameter $\sim 110 \mathrm{~m}$, distances between neighbouring turbines $0.6 \sim 1.2 \mathrm{~km}$ ) have been established within and near the wetland since 2013 (Fig. 1b), which might disturb the bird community in the area.

\section{Bird survey}

We used point counts to investigate the bird community in the Rudong coastal wetland (Ralph et al., 1995; Bibby et al., 2000). A total of 40 census points were randomly established in the wetland (Fig. 1b); the points were separated by a distance of at least $400 \mathrm{~m}$. The counting radius and duration at each point were $100 \mathrm{~m}$ and $5 \mathrm{~min}$, respectively (Ralph et al., 1995; Bibby et al., 2000). Our surveys were carried out in 2019 and 2020. Because the bird migration peak in East China's coastal wetlands occurs in spring and autumn (Ma et al., 2009), the counts were performed from March to May (spring) and from September to November (autumn) in the two years. The counts were conducted in the first 3 hours after dawn and at middle or low tide under fair weather conditions. The point counts were repeated 10 times per season per year. Each time 4, teams of trained observers ( 2 observers per team) visited the points (10 points for each team). All birds seen or heard around the points were recorded. Binoculars (Nature DX ED 10×50, Celestron, USA) were used for observation. Bird flocks were recorded by cameras (EOS 700D, Canon, Japan) with spotting scopes (Ultima 80, Celestron, USA) and were counted after the surveys. For each season, the point count result was obtained by summing the 20 replicates over the two years. The total number of a species was the sum of its numbers at the 40 points.

\section{Data preparation and analysis}

In this study, we divided species dominance into 4 classes according to the proportion (F) of the total number of a species to that of all species, i.e., dominant species $(F>5 \%)$, subdominant species $(2 \%<F \leq 5 \%)$, occasional species $(1 \%<F \leq$ $2 \%)$, and rare species ( $F \leq 1 \%)$ (Lenz, 1990). Moreover, we divided the recorded birds into two general categories. Birds of Charadriiformes, Ciconiiformes, Anseriformes, Gruiformes, and Podicipediformes were all regarded as aquatic birds, whereas those of Passeriformes, Falconiformes, Coraciiformes, Columbiformes, Cuculiformes, and Galliformes were regarded as terrestrial birds.

To investigate the spatial responses of the bird community to the wind farm, we determined the distance between each census point and the wind farm boundary (DW) in Fig. 1b. DW was given a negative value if the point was located within 
the wind farm. Moreover, in this study, we considered 3 other geographical factors at the census points that influenced bird distributions (Niemuth et al., 2006; Chapman and Reich, 2007; Ma and Chen, 2018), i.e., the distance between each census point and the suburbs (DR), the distance between each census point and the sea (DS), and the vegetation area surrounding (100 m radius) each census point (VA). DR, DS, and VA were also determined in Fig. 1b and were treated as controlled factors in partial correlation analysis.

Partial correlation analysis was used to examine the relationship between bird number and DW; DR, DS, and VA were all regarded as controlled factors. A positive correlation indicated that the number increased with $D W$, whereas a negative correlation indicated a decrease. Furthermore, redundancy analysis (RDA) was conducted to sketch the responses of the bird community to the 4 factors. The point count results were standardized by Hellinger transformation before RDA (Borcard et al., 2011). To show the RDA results more clearly, bird species were divided into Groups A and B in each season. Species in Group A had higher RDA scores, and their scores were magnified by 2.5 times; those in Group B had lower RDA scores, and the scores were magnified by 15 times. Moreover, the census points are not shown in the plots because the geographical factors at each point can be found in Fig. 1b. Finally, stepwise regression was used to describe the variation of bird number with DW as well as other 3 factors. Factors were eliminated from the regression equations when their significance levels were higher than 0.10 . The partial correlation analysis, RDA, and stepwise regression were performed in RStudio 1.3.959.

\section{Results}

A total of 52,571 birds of 11 orders and 103 species were counted during the survey. A total of 30,609 birds of 88 species were recorded in spring, while 21,962 birds of 78 species were recorded in autumn (Table 1). The total count and species richness were all higher in spring.

Charadriiformes was the dominant order in the wetland, comprising 43 species and $95.8 \%$ of the total count over the two seasons. Passeriformes comprised 39 species, which was the second highest richness. However, the passerines in the community were mostly rare species, and they comprised only $2.7 \%$ of the total count. Other recorded bird orders included Ciconiiformes, Anseriformes, Gruiformes, Podicipediformes, Coraciiformes, Columbiformes, Cuculiformes, Falconiformes, and Galliformes. These 9 orders had low species richnesses and bird numbers; they comprised only 21 species and only $1.5 \%$ of the total count.

Calidris alpina was the most common species in both spring and autumn. It comprised $18.5 \%$ and $24.0 \%$ of the total counts in spring and autumn, respectively. Other dominant species included Calidris ruficollis, Charadrius alexandrines, Calidris tenuirostris, and Tringa nebularia (spring). The subdominant species included Xenus cinereus, Numenius phaeopus, Calidris acuminata, Charadrius mongolus, Charadrius leschenaultii, Calidris canutus (spring), Arenaria interpres (spring), Pluvialis squatarola (spring), Tringa nebularia (autumn), and Chroicocephalus saundersi (autumn).

According to the IUCN Red List of threatened species (IUCN, 2021), 10 of the 103 recorded species are near threatened, 3 of them are vulnerable, 3 are endangered, and 1 is critically endangered. The 3 endangered species were Calidris tenuirostris, Numenius madagascariensis, and Platalea minor. Calidris pygmaea was the only critically endangered species. 
Table 1

Composition of the bird community in the Rudong coastal wetland.

\begin{tabular}{|c|c|c|c|c|c|c|c|c|}
\hline \multirow{2}{*}{$\begin{array}{l}\text { Species } \\
\text { number } \\
\text { a }\end{array}$} & \multicolumn{4}{|c|}{ Spring } & \multicolumn{4}{|c|}{ Autumn } \\
\hline & $\begin{array}{l}\text { Bird } \\
\text { order } \\
\text { b }\end{array}$ & Scientific name $^{c}$ & $\begin{array}{l}\text { Total } \\
\text { number } \\
d\end{array}$ & $\begin{array}{l}\text { Partial } \\
\text { correlation } \\
\text { coefficient } \\
\text { e }\end{array}$ & $\begin{array}{l}\text { Bird } \\
\text { order } \\
\text { b }\end{array}$ & Scientific name $^{c}$ & $\begin{array}{l}\text { Total } \\
\text { number } \\
d\end{array}$ & $\begin{array}{l}\text { Partial } \\
\text { correlation } \\
\text { coefficient } \\
\text { e }\end{array}$ \\
\hline 1 & Cha. & Calidris alpina ${ }^{(\mathrm{NT})}$ & $\begin{array}{l}5652 \\
+++\end{array}$ & 0.581 & Cha. & Calidris alpina & $\begin{array}{l}5272 \\
+++\end{array}$ & 0.735 \\
\hline 2 & & Calidris ruficollis & $\begin{array}{l}4808 \\
+++\end{array}$ & 0.319 & & $\begin{array}{l}\text { Charadrius } \\
\text { alexandrinus }\end{array}$ & $\begin{array}{l}3626 \\
+++\end{array}$ & 0.434 \\
\hline 3 & & $\begin{array}{l}\text { Charadrius } \\
\text { alexandrinus }\end{array}$ & $\begin{array}{l}3780 \\
+++\end{array}$ & 0.398 & & $\begin{array}{l}\text { Calidris ruficollis } \\
\text { (NT) }\end{array}$ & $\begin{array}{l}3135 \\
+++\end{array}$ & 0.524 \\
\hline 4 & & $\begin{array}{l}\text { Calidris } \\
\text { tenuirostris }(\mathrm{EN})\end{array}$ & $\begin{array}{l}2226 \\
+++\end{array}$ & 0.530 & & $\begin{array}{l}\text { Calidris } \\
\text { tenuirostris }(\mathrm{EN})\end{array}$ & $\begin{array}{l}1404 \\
+++\end{array}$ & 0.467 \\
\hline 5 & & Tringa nebularia & $\begin{array}{l}1803 \\
+++\end{array}$ & 0.459 & & $\begin{array}{l}\text { Calidris } \\
\text { acuminata }\end{array}$ & $1026^{++}$ & 0.365 \\
\hline 6 & & Xenus cinereus & $1468^{++}$ & 0.425 & & Xenus cinereus & $912^{++}$ & 0.425 \\
\hline 7 & & $\begin{array}{l}\text { Numenius } \\
\text { phaeopus }\end{array}$ & $1073^{++}$ & -0.094 & & Tringa nebularia & $745^{++}$ & 0.262 \\
\hline 8 & & $\begin{array}{l}\text { Calidris } \\
\text { acuminata }\end{array}$ & $987^{++}$ & 0.527 & & $\begin{array}{l}\text { Numenius } \\
\text { phaeopus }\end{array}$ & $664^{++}$ & -0.112 \\
\hline 9 & & $\begin{array}{l}\text { Charadrius } \\
\text { mongolus }\end{array}$ & $978^{++}$ & 0.227 & & $\begin{array}{l}\text { Charadrius } \\
\text { leschenaultii }\end{array}$ & $602^{++}$ & 0.201 \\
\hline 10 & & $\begin{array}{l}\text { Calidris canutus } \\
\text { (NT) }\end{array}$ & $822^{++}$ & -0.063 & & $\begin{array}{l}\text { Charadrius } \\
\text { mongolus }\end{array}$ & $541^{++}$ & 0.534 \\
\hline 11 & & $\begin{array}{l}\text { Charadrius } \\
\text { leschenaultii }\end{array}$ & $816^{++}$ & 0.308 & & $\begin{array}{l}\text { Chroicocephalus } \\
\text { saundersi }(\mathrm{VU})\end{array}$ & $469^{++}$ & 0.303 \\
\hline 12 & & Arenaria interpres & $789^{++}$ & -0.196 & & $\begin{array}{l}\text { Calidris canutus } \\
\text { (NT) }\end{array}$ & $422^{+}$ & 0.190 \\
\hline
\end{tabular}

a The dominant, subdominant, and occasional species are arranged according to total bird number. The rare species are arranged according to taxonomy.

${ }^{b}$ Cha.: Charadriiformes; Pas.: Passeriformes; Gal.: Galliformes; Gru.: Gruiformes; Ans.: Anseriformes; Pod.:

Podicipediformes; Cic.: Ciconiiformes; Cor.: Coraciiformes; Col.: Columbiformes; Cuc.: Cuculiformes; Fal.:

Falconiformes.

c The superscripts with brackets represent the endangered categories according to IUCN (2021). NT: near threatened; VU: vulnerable; EN: endangered; CR: critically endangered. The species of least concern have no superscript.

d The superscripts +++, ++, and + indicate dominant species, subdominant species, and occasional species, respectively. Rare species have no superscript.

e The partial correlation coefficient between bird number and DW. A positive value indicates that the bird number increased with DW, whereas a negative value indicates a decrease. Values in bold indicate significant correlations at the $p<0.05$ level. 


\begin{tabular}{|c|c|c|c|c|c|c|c|c|}
\hline \multirow{2}{*}{$\begin{array}{l}\text { Species } \\
\text { number } \\
\text { a }\end{array}$} & \multicolumn{4}{|c|}{ Spring } & \multicolumn{4}{|c|}{ Autumn } \\
\hline & $\begin{array}{l}\text { Bird } \\
\text { order } \\
\text { b }\end{array}$ & Scientific name $^{c}$ & $\begin{array}{l}\text { Total } \\
\text { number } \\
\text { d }\end{array}$ & $\begin{array}{l}\text { Partial } \\
\text { correlation } \\
\text { coefficient } \\
\text { e }\end{array}$ & $\begin{array}{l}\text { Bird } \\
\text { order } \\
\text { b }\end{array}$ & Scientific name $^{c}$ & $\begin{array}{l}\text { Total } \\
\text { number } \\
\text { d }\end{array}$ & $\begin{array}{l}\text { Partial } \\
\text { correlation } \\
\text { coefficient } \\
\text { e }\end{array}$ \\
\hline 13 & & $\begin{array}{l}\text { Pluvialis } \\
\text { squatarola }\end{array}$ & $657^{++}$ & 0.393 & & $\begin{array}{l}\text { Limosa lapponica } \\
\text { (NT) }\end{array}$ & $396^{+}$ & 0.207 \\
\hline 14 & & $\begin{array}{l}\text { Numenius } \\
\operatorname{arquata}^{(\mathrm{NT})}\end{array}$ & $617^{+}$ & -0.308 & Pas. & Passer montanus & $327^{+}$ & -0.399 \\
\hline 15 & & $\begin{array}{l}\text { Limosa lapponica } \\
\text { (NT) }\end{array}$ & $556^{+}$ & -0.229 & Cha. & $\begin{array}{l}\text { Numenius } \\
\operatorname{arquata}^{(\mathrm{NT})}\end{array}$ & $236^{+}$ & -0.067 \\
\hline 16 & & $\begin{array}{l}\text { Chroicocephalus } \\
\text { saundersi }(\mathrm{VU})\end{array}$ & $467^{+}$ & 0.046 & & Arenaria interpres & 180 & 0.211 \\
\hline 17 & & $\begin{array}{l}\text { Tringa brevipes } \\
\text { (NT) }\end{array}$ & $382^{+}$ & 0.254 & & Calidris alba & 4 & -0.398 \\
\hline 18 & & Calidris alba & 34 & -0.320 & & $\begin{array}{l}\text { Calidris ferruginea } \\
\text { (NT) }\end{array}$ & 27 & -0.233 \\
\hline 19 & & $\begin{array}{l}\text { Calidris ferruginea } \\
\text { (NT) }\end{array}$ & 34 & -0.284 & & $\begin{array}{l}\text { Calidris pygmaea } \\
\text { (CR) }\end{array}$ & 8 & -0.137 \\
\hline 20 & & $\begin{array}{l}\text { Calidris } \\
\text { melanotos }\end{array}$ & 26 & -0.005 & & $\begin{array}{l}\text { Calidris } \\
\text { subminuta }\end{array}$ & 13 & -0.551 \\
\hline 21 & & $\begin{array}{l}\text { Calidris } \\
\text { subminuta }\end{array}$ & 12 & -0.256 & & $\begin{array}{l}\text { Chroicocephalus } \\
\text { ridibundus }\end{array}$ & 179 & -0.200 \\
\hline 22 & & $\begin{array}{l}\text { Chroicocephalus } \\
\text { ridibundus }\end{array}$ & 197 & 0.115 & & $\begin{array}{l}\text { Gelochelidon } \\
\text { nilotica }\end{array}$ & 48 & 0.091 \\
\hline 23 & & $\begin{array}{l}\text { Gallinago } \\
\text { gallinago }\end{array}$ & 7 & -0.091 & & $\begin{array}{l}\text { Haematopus } \\
\text { ostralegus }^{(\mathrm{NT})}\end{array}$ & 3 & 0.036 \\
\hline 24 & & $\begin{array}{l}\text { Gelochelidon } \\
\text { nilotica }\end{array}$ & 80 & -0.157 & & $\begin{array}{l}\text { Himantopus } \\
\text { himantopus }\end{array}$ & 2 & -0.070 \\
\hline 25 & & $\begin{array}{l}\text { Haematopus } \\
\text { ostralegus }^{(\mathrm{NT})}\end{array}$ & 9 & 0.032 & & Larus canus & 62 & 0.061 \\
\hline
\end{tabular}

a The dominant, subdominant, and occasional species are arranged according to total bird number. The rare species are arranged according to taxonomy.

${ }^{b}$ Cha.: Charadriiformes; Pas.: Passeriformes; Gal.: Galliformes; Gru.: Gruiformes; Ans.: Anseriformes; Pod.: Podicipediformes; Cic.: Ciconiiformes; Cor.: Coraciiformes; Col.: Columbiformes; Cuc.: Cuculiformes; Fal.: Falconiformes.

${ }^{c}$ The superscripts with brackets represent the endangered categories according to IUCN (2021). NT: near threatened; VU: vulnerable; EN: endangered; CR: critically endangered. The species of least concern have no superscript.

d The superscripts,+++++ , and + indicate dominant species, subdominant species, and occasional species, respectively. Rare species have no superscript.

e The partial correlation coefficient between bird number and DW. A positive value indicates that the bird number increased with DW, whereas a negative value indicates a decrease. Values in bold indicate significant correlations at the $p<0.05$ level. 


\begin{tabular}{|c|c|c|c|c|c|c|c|c|}
\hline \multirow{2}{*}{$\begin{array}{l}\text { Species } \\
\text { number } \\
\text { a }\end{array}$} & \multicolumn{4}{|l|}{ Spring } & \multicolumn{4}{|c|}{ Autumn } \\
\hline & $\begin{array}{l}\text { Bird } \\
\text { order } \\
\text { b }\end{array}$ & Scientific name $^{c}$ & $\begin{array}{l}\text { Total } \\
\text { number } \\
\text { d }\end{array}$ & $\begin{array}{l}\text { Partial } \\
\text { correlation } \\
\text { coefficient } \\
\text { e }\end{array}$ & $\begin{array}{l}\text { Bird } \\
\text { order } \\
\text { b }\end{array}$ & Scientific name $^{c}$ & $\begin{array}{l}\text { Total } \\
\text { number } \\
\text { d }\end{array}$ & $\begin{array}{l}\text { Partial } \\
\text { correlation } \\
\text { coefficient } \\
\text { e }\end{array}$ \\
\hline 26 & & $\begin{array}{l}\text { Himantopus } \\
\text { himantopus }\end{array}$ & 9 & -0.078 & & Larus crassirostris & 14 & -0.360 \\
\hline 27 & & Larus canus & 58 & 0.127 & & Larus mongolicus & 133 & 0.019 \\
\hline 28 & & Larus crassirostris & 17 & -0.432 & & $\begin{array}{l}\text { Limosa limosa } \\
\text { (NT) }\end{array}$ & 40 & -0.213 \\
\hline 29 & & Larus mongolicus & 97 & 0.422 & & $\begin{array}{l}\text { Numenius } \\
\text { madagascariensis } \\
\text { (EN) }\end{array}$ & 162 & -0.040 \\
\hline 30 & & $\begin{array}{l}\text { Limicola } \\
\text { falcinellus }\end{array}$ & 18 & -0.116 & & $\begin{array}{l}\text { Pluvialis } \\
\text { squatarola }\end{array}$ & 153 & -0.187 \\
\hline 31 & & $\begin{array}{l}\text { Limosa limosa } \\
\text { (NT) }\end{array}$ & 268 & -0.099 & & $\begin{array}{l}\text { Recurvirostra } \\
\text { avosetta }\end{array}$ & 24 & 0.051 \\
\hline 32 & & $\begin{array}{l}\text { Numenius } \\
\text { madagascariensis } \\
\text { (NT) }\end{array}$ & 15 & 0.139 & & Sterna hirundo & 21 & -0.089 \\
\hline 33 & & Pluvialis fulva & 216 & -0.172 & & Sternula albifrons & 9 & 0.012 \\
\hline 34 & & $\begin{array}{l}\text { Recurvirostra } \\
\text { avosetta }\end{array}$ & 18 & -0.278 & & $\begin{array}{l}\text { Tringa brevipes } \\
\text { (NT) }\end{array}$ & 100 & -0.211 \\
\hline 35 & & Sterna hirundo & 14 & 0.056 & & Tringa erythropus & 7 & 0.102 \\
\hline 36 & & Sternula albifrons & 45 & -0.088 & & Tringa glareola & 210 & -0.206 \\
\hline 37 & & Tringa erythropus & 21 & -0.082 & & Tringa hypoleucos & 20 & -0.202 \\
\hline 38 & & Tringa glareola & 42 & -0.326 & & Tringa stagnatilis & 18 & 0.080 \\
\hline 39 & & Tringa hypoleucos & 16 & -0.080 & & Tringa totanus & 19 & -0.059 \\
\hline 40 & & Tringa totanus & 299 & 0.086 & Cic. & Ardea alba & 10 & 0.117 \\
\hline 41 & & Vanellus cinereus & 2 & 0.025 & & Ardea cinerea & 7 & -0.131 \\
\hline \multicolumn{9}{|c|}{$\begin{array}{l}\text { a The dominant, subdominant, and occasional species are arranged according to total bird number. The rare species } \\
\text { are arranged according to taxonomy. }\end{array}$} \\
\hline \multicolumn{9}{|c|}{$\begin{array}{l}\text { b Cha.: Charadriiformes; Pas.: Passeriformes; Gal.: Galliformes; Gru.: Gruiformes; Ans.: Anseriformes; Pod.: } \\
\text { Podicipediformes; Cic.: Ciconiiformes; Cor.: Coraciiformes; Col.: Columbiformes; Cuc.: Cuculiformes; Fal.: } \\
\text { Falconiformes. }\end{array}$} \\
\hline \multicolumn{9}{|c|}{$\begin{array}{l}\text { c The superscripts with brackets represent the endangered categories according to IUCN (2021). NT: near threatened; } \\
\text { VU: vulnerable; EN: endangered; CR: critically endangered. The species of least concern have no superscript. }\end{array}$} \\
\hline \multicolumn{9}{|c|}{$\begin{array}{l}\text { d The superscripts }+++,++ \text {, and }+ \text { indicate dominant species, subdominant species, and occasional species, } \\
\text { respectively. Rare species have no superscript. }\end{array}$} \\
\hline \multicolumn{9}{|c|}{$\begin{array}{l}\text { e The partial correlation coefficient between bird number and DW. A positive value indicates that the bird number } \\
\text { increased with DW, whereas a negative value indicates a decrease. Values in bold indicate significant correlations at } \\
\text { the } p<0.05 \text { level. }\end{array}$} \\
\hline
\end{tabular}




\begin{tabular}{|c|c|c|c|c|c|c|c|c|}
\hline \multirow{2}{*}{$\begin{array}{l}\text { Species } \\
\text { number } \\
\text { a }\end{array}$} & \multicolumn{4}{|c|}{ Spring } & \multicolumn{4}{|c|}{ Autumn } \\
\hline & $\begin{array}{l}\text { Bird } \\
\text { order } \\
\text { b }\end{array}$ & Scientific name $^{c}$ & $\begin{array}{l}\text { Total } \\
\text { number } \\
d\end{array}$ & $\begin{array}{l}\text { Partial } \\
\text { correlation } \\
\text { coefficient } \\
\text { e }\end{array}$ & $\begin{array}{l}\text { Bird } \\
\text { order } \\
\text { b }\end{array}$ & Scientific name $^{c}$ & $\begin{array}{l}\text { Total } \\
\text { number } \\
d\end{array}$ & $\begin{array}{l}\text { Partial } \\
\text { correlation } \\
\text { coefficient } \\
\text { e }\end{array}$ \\
\hline 42 & \multirow[t]{6}{*}{ Cic. } & Ardea alba & 28 & -0.236 & & Bubulcus ibis & 8 & 0.010 \\
\hline 43 & & Ardea cinerea & 26 & 0.101 & & Egretta garzetta & 195 & 0.036 \\
\hline 44 & & Bubulcus ibis & 8 & 0.028 & & Egretta intermedia & 18 & 0.081 \\
\hline 45 & & Egretta garzetta & 168 & -0.170 & \multirow[t]{4}{*}{ Ans. } & Anas falcata ${ }^{(\mathrm{NT})}$ & 8 & -0.083 \\
\hline 46 & & Egretta intermedia & 108 & -0.185 & & Anas penelope & 16 & 0.091 \\
\hline 47 & & $\begin{array}{l}\text { Platalea minor } \\
\text { (EN) }\end{array}$ & 8 & -0.375 & & $\begin{array}{l}\text { Anas } \\
\text { platyrhynchos }\end{array}$ & 20 & 0.141 \\
\hline 48 & \multirow[t]{4}{*}{ Ans. } & Anas falcata (NT) & 23 & 0.174 & & $\begin{array}{l}\text { Anas } \\
\text { zonorhyncha }\end{array}$ & 15 & 0.117 \\
\hline 49 & & $\begin{array}{l}\text { Anas } \\
\text { platyrhynchos }\end{array}$ & 37 & -0.151 & \multirow[t]{2}{*}{ Gru. } & Porzana pusilla & 6 & -0.338 \\
\hline 50 & & Anas querquedula & 9 & 0.171 & & Rallus aquaticus & 1 & 0.115 \\
\hline 51 & & $\begin{array}{l}\text { Anas } \\
\text { zonorhyncha }\end{array}$ & 31 & -0.230 & \multirow[t]{7}{*}{ Pas. } & $\begin{array}{l}\text { Tachybaptus } \\
\text { ruficollis }\end{array}$ & 6 & -0.107 \\
\hline 52 & \multirow[t]{3}{*}{ Gru. } & Fulica atra & 6 & -0.137 & & $\begin{array}{l}\text { Acrocephalus } \\
\text { bistrigiceps }\end{array}$ & 4 & -0.170 \\
\hline 53 & & Porzana pusilla & 4 & 0.035 & & $\begin{array}{l}\text { Acrocephalus } \\
\text { tangorum }^{(\mathrm{VU})}\end{array}$ & 1 & 0.042 \\
\hline 54 & & Rallus aquaticus & 3 & -0.143 & & Anthus richardi & 7 & -0.082 \\
\hline 55 & Pod. & $\begin{array}{l}\text { Tachybaptus } \\
\text { ruficollis }\end{array}$ & 7 & -0.227 & & Cecropis daurica & 22 & -0.165 \\
\hline 56 & \multirow[t]{2}{*}{ Pas. } & $\begin{array}{l}\text { Acrocephalus } \\
\text { orientalis }\end{array}$ & 4 & -0.222 & & Cisticola juncidis & 3 & 0.040 \\
\hline 57 & & Alauda gulgula & 4 & 0.258 & & Cyanopica cyanus & 42 & -0.220 \\
\hline
\end{tabular}

a The dominant, subdominant, and occasional species are arranged according to total bird number. The rare species are arranged according to taxonomy.

${ }^{b}$ Cha.: Charadriiformes; Pas.: Passeriformes; Gal.: Galliformes; Gru.: Gruiformes; Ans.: Anseriformes; Pod.: Podicipediformes; Cic.: Ciconiiformes; Cor.: Coraciiformes; Col.: Columbiformes; Cuc.: Cuculiformes; Fal.: Falconiformes.

${ }^{c}$ The superscripts with brackets represent the endangered categories according to IUCN (2021). NT: near threatened; VU: vulnerable; EN: endangered; CR: critically endangered. The species of least concern have no superscript.

d The superscripts,+++++ , and + indicate dominant species, subdominant species, and occasional species, respectively. Rare species have no superscript.

e The partial correlation coefficient between bird number and DW. A positive value indicates that the bird number increased with DW, whereas a negative value indicates a decrease. Values in bold indicate significant correlations at the $p<0.05$ level. 


\begin{tabular}{|c|c|c|c|c|c|c|c|c|}
\hline \multirow{2}{*}{$\begin{array}{l}\text { Species } \\
\text { number } \\
\text { a }\end{array}$} & \multicolumn{4}{|l|}{ Spring } & \multicolumn{4}{|c|}{ Autumn } \\
\hline & $\begin{array}{l}\text { Bird } \\
\text { order } \\
\text { b }\end{array}$ & Scientific name $^{c}$ & $\begin{array}{l}\text { Total } \\
\text { number } \\
\text { d }\end{array}$ & $\begin{array}{l}\text { Partial } \\
\text { correlation } \\
\text { coefficient } \\
\text { e }\end{array}$ & $\begin{array}{l}\text { Bird } \\
\text { order } \\
\text { b }\end{array}$ & Scientific name $^{c}$ & $\begin{array}{l}\text { Total } \\
\text { number } \\
\text { d }\end{array}$ & $\begin{array}{l}\text { Partial } \\
\text { correlation } \\
\text { coefficient } \\
\text { e }\end{array}$ \\
\hline 58 & & Cecropis daurica & 28 & -0.314 & & Emberiza pallasi & 6 & -0.099 \\
\hline 59 & & Cisticola juncidis & 6 & -0.151 & & Emberiza pusilla & 14 & 0.042 \\
\hline 60 & & Cyanopica cyanus & 25 & -0.127 & & $\begin{array}{l}\text { Ficedula } \\
\text { mugimaki }\end{array}$ & 4 & -0.176 \\
\hline 61 & & Emberiza cioides & 17 & 0.172 & & $\begin{array}{l}\text { Fringilla } \\
\text { montifringilla }\end{array}$ & 14 & -0.208 \\
\hline 62 & & Emberiza rutila & 15 & -0.084 & & Hirundo rustica & 21 & -0.049 \\
\hline 63 & & $\begin{array}{l}\text { Ficedula } \\
\text { mugimaki }\end{array}$ & 6 & -0.251 & & Lanius schach & 18 & -0.226 \\
\hline 64 & & $\begin{array}{l}\text { Ficedula } \\
\text { zanthopygia }\end{array}$ & 6 & 0.073 & & Luscinia calliope & 7 & 0.186 \\
\hline 65 & & $\begin{array}{l}\text { Fringilla } \\
\text { montifringilla }\end{array}$ & 40 & -0.274 & & Luscinia cyane & 1 & -0.177 \\
\hline 66 & & Hirundo rustica & 61 & -0.332 & & $\begin{array}{l}\text { Motacilla } \\
\text { tschutschensis }\end{array}$ & 9 & -0.176 \\
\hline 67 & & Lanius schach & 22 & -0.194 & & $\begin{array}{l}\text { Muscicapa } \\
\text { griseisticta }\end{array}$ & 20 & -0.381 \\
\hline 68 & & $\begin{array}{l}\text { Locustella } \\
\text { lanceolata }\end{array}$ & 4 & -0.260 & & $\begin{array}{l}\text { Muscicapa } \\
\text { latirostris }\end{array}$ & 5 & -0.125 \\
\hline 69 & & $\begin{array}{l}\text { Locustella pleskei } \\
(\mathrm{VU})\end{array}$ & 8 & 0.231 & & $\begin{array}{l}\text { Paradoxornis } \\
\text { heudei }^{(\mathrm{NT})}\end{array}$ & 69 & -0.236 \\
\hline 70 & & Luscinia calliope & 4 & -0.178 & & $\begin{array}{l}\text { Phylloscopus } \\
\text { borealis }\end{array}$ & 8 & -0.014 \\
\hline 71 & & $\begin{array}{l}\text { Motacilla } \\
\text { tschutschensis }\end{array}$ & 10 & -0.155 & & $\begin{array}{l}\text { Phylloscopus } \\
\text { coronatus }\end{array}$ & 3 & -0.122 \\
\hline 72 & & $\begin{array}{l}\text { Muscicapa } \\
\text { griseisticta }\end{array}$ & 14 & -0.192 & & $\begin{array}{l}\text { Phylloscopus } \\
\text { tenellipes }\end{array}$ & 8 & 0.200 \\
\hline \multicolumn{9}{|c|}{$\begin{array}{l}\text { a The dominant, subdominant, and occasional species are arranged according to total bird number. The rare species } \\
\text { are arranged according to taxonomy. }\end{array}$} \\
\hline \multicolumn{9}{|c|}{$\begin{array}{l}\text { b Cha.: Charadriiformes; Pas.: Passeriformes; Gal.: Galliformes; Gru.: Gruiformes; Ans.: Anseriformes; Pod.: } \\
\text { Podicipediformes; Cic.: Ciconiiformes; Cor.: Coraciiformes; Col.: Columbiformes; Cuc.: Cuculiformes; Fal.: } \\
\text { Falconiformes. }\end{array}$} \\
\hline \multicolumn{9}{|c|}{$\begin{array}{l}\text { c The superscripts with brackets represent the endangered categories according to IUCN (2021). NT: near threatened; } \\
\text { VU: vulnerable; EN: endangered; CR: critically endangered. The species of least concern have no superscript. }\end{array}$} \\
\hline \multicolumn{9}{|c|}{$\begin{array}{l}\text { d The superscripts }+++,++ \text {, and }+ \text { indicate dominant species, subdominant species, and occasional species, } \\
\text { respectively. Rare species have no superscript. }\end{array}$} \\
\hline \multicolumn{9}{|c|}{$\begin{array}{l}\text { e The partial correlation coefficient between bird number and DW. A positive value indicates that the bird number } \\
\text { increased with DW, whereas a negative value indicates a decrease. Values in bold indicate significant correlations at } \\
\text { the } p<0.05 \text { level. }\end{array}$} \\
\hline
\end{tabular}




\begin{tabular}{|c|c|c|c|c|c|c|c|c|}
\hline \multirow{2}{*}{$\begin{array}{l}\text { Species } \\
\text { number } \\
\text { a }\end{array}$} & \multicolumn{4}{|l|}{ Spring } & \multicolumn{4}{|c|}{ Autumn } \\
\hline & $\begin{array}{l}\text { Bird } \\
\text { order } \\
\text { b }\end{array}$ & Scientific name $^{c}$ & $\begin{array}{l}\text { Total } \\
\text { number } \\
\text { d }\end{array}$ & $\begin{array}{l}\text { Partial } \\
\text { correlation } \\
\text { coefficient } \\
\text { e }\end{array}$ & $\begin{array}{l}\text { Bird } \\
\text { order } \\
\text { b }\end{array}$ & Scientific name $^{c}$ & $\begin{array}{l}\text { Total } \\
\text { number } \\
\text { d }\end{array}$ & $\begin{array}{l}\text { Partial } \\
\text { correlation } \\
\text { coefficient } \\
\text { e }\end{array}$ \\
\hline 73 & & $\begin{array}{l}\text { Paradoxornis } \\
\text { heudei }^{(\mathrm{NT})}\end{array}$ & 36 & -0.151 & & $\begin{array}{l}\text { Pycnonotus } \\
\text { sinensis }\end{array}$ & 10 & 0.088 \\
\hline 74 & & $\begin{array}{l}\text { Paradoxornis } \\
\text { webbianus }\end{array}$ & 8 & -0.292 & & $\begin{array}{l}\text { Remiz } \\
\text { consobrinus }\end{array}$ & 98 & -0.350 \\
\hline 75 & & Parus major & 10 & -0.173 & & $\begin{array}{l}\text { Turdus } \\
\text { hortulorum }\end{array}$ & 6 & 0.028 \\
\hline 76 & & Passer montanus & 225 & -0.216 & & Turdus obscurus & 8 & -0.078 \\
\hline 77 & & $\begin{array}{l}\text { Phoenicurus } \\
\text { auroreus }\end{array}$ & 8 & -0.244 & Cor. & Halcyon pileata & 1 & 0.299 \\
\hline 78 & & $\begin{array}{l}\text { Phylloscopus } \\
\text { coronatus }\end{array}$ & 6 & -0.239 & Gal. & $\begin{array}{l}\text { Phasianus } \\
\text { colchicus }\end{array}$ & 10 & -0.153 \\
\hline 79 & & $\begin{array}{l}\text { Phylloscopus } \\
\text { inornatus }\end{array}$ & 20 & -0.302 & & & & \\
\hline 80 & & $\begin{array}{l}\text { Phylloscopus } \\
\text { tenellipes }\end{array}$ & 20 & 0.033 & & & & \\
\hline 81 & & $\begin{array}{l}\text { Pycnonotus } \\
\text { sinensis }\end{array}$ & 32 & -0.346 & & & & \\
\hline 82 & & $\begin{array}{l}\text { Remiz } \\
\text { consobrinus }\end{array}$ & 48 & -0.149 & & & & \\
\hline 83 & & $\begin{array}{l}\text { Spodiopsar } \\
\text { sericeus }\end{array}$ & 7 & -0.075 & & & & \\
\hline 84 & & Turdus cardis & 9 & -0.195 & & & & \\
\hline 85 & Cor. & Upupa epops & 2 & -0.272 & & & & \\
\hline 86 & Col. & $\begin{array}{l}\text { Spilopelia } \\
\text { chinensis }\end{array}$ & 1 & -0.118 & & & & \\
\hline 87 & Cuc. & $\begin{array}{l}\text { Cuculus } \\
\text { poliocephalus }\end{array}$ & 1 & -0.054 & & & & \\
\hline \multicolumn{9}{|c|}{$\begin{array}{l}\text { a The dominant, subdominant, and occasional species are arranged according to total bird number. The rare species } \\
\text { are arranged according to taxonomy. }\end{array}$} \\
\hline \multicolumn{9}{|c|}{$\begin{array}{l}\text { b Cha.: Charadriiformes; Pas.: Passeriformes; Gal.: Galliformes; Gru.: Gruiformes; Ans.: Anseriformes; Pod.: } \\
\text { Podicipediformes; Cic.: Ciconiiformes; Cor.: Coraciiformes; Col.: Columbiformes; Cuc.: Cuculiformes; Fal.: } \\
\text { Falconiformes. }\end{array}$} \\
\hline \multicolumn{9}{|c|}{$\begin{array}{l}\text { c The superscripts with brackets represent the endangered categories according to IUCN (2021). NT: near threatened; } \\
\text { VU: vulnerable; EN: endangered; CR: critically endangered. The species of least concern have no superscript. }\end{array}$} \\
\hline \multicolumn{9}{|c|}{$\begin{array}{l}\text { d The superscripts }+++,++ \text {, and }+ \text { indicate dominant species, subdominant species, and occasional species, } \\
\text { respectively. Rare species have no superscript. }\end{array}$} \\
\hline \multicolumn{9}{|c|}{$\begin{array}{l}\text { e The partial correlation coefficient between bird number and DW. A positive value indicates that the bird number } \\
\text { increased with DW, whereas a negative value indicates a decrease. Values in bold indicate significant correlations at } \\
\text { the } p<0.05 \text { level. }\end{array}$} \\
\hline
\end{tabular}




\begin{tabular}{|c|c|c|c|c|c|c|c|c|}
\hline \multirow{2}{*}{$\begin{array}{l}\text { Species } \\
\text { number } \\
\text { a }\end{array}$} & \multicolumn{4}{|c|}{ Spring } & \multicolumn{4}{|c|}{ Autumn } \\
\hline & $\begin{array}{l}\text { Bird } \\
\text { order } \\
\text { b }\end{array}$ & Scientific name $^{c}$ & $\begin{array}{l}\text { Total } \\
\text { number } \\
\text { d }\end{array}$ & $\begin{array}{l}\text { Partial } \\
\text { correlation } \\
\text { coefficient } \\
\text { e }\end{array}$ & $\begin{array}{l}\text { Bird } \\
\text { order } \\
\text { b }\end{array}$ & Scientific name $^{c}$ & $\begin{array}{l}\text { Total } \\
\text { number } \\
\text { d }\end{array}$ & $\begin{array}{l}\text { Partial } \\
\text { correlation } \\
\text { coefficient } \\
\text { e }\end{array}$ \\
\hline 88 & Fal. & Falco tinnunculus & 1 & 0.299 & & & & \\
\hline Total & & & 30609 & & & & 21962 & \\
\hline \multicolumn{9}{|c|}{$\begin{array}{l}\text { a The dominant, subdominant, and occasional species are arranged according to total bird number. The rare species } \\
\text { are arranged according to taxonomy. }\end{array}$} \\
\hline \multicolumn{9}{|c|}{$\begin{array}{l}\text { b Cha.: Charadriiformes; Pas.: Passeriformes; Gal.: Galliformes; Gru.: Gruiformes; Ans.: Anseriformes; Pod.: } \\
\text { Podicipediformes; Cic.: Ciconiiformes; Cor.: Coraciiformes; Col.: Columbiformes; Cuc.: Cuculiformes; Fal.: } \\
\text { Falconiformes. }\end{array}$} \\
\hline \multicolumn{9}{|c|}{$\begin{array}{l}\text { c The superscripts with brackets represent the endangered categories according to IUCN (2021). NT: near threatened; } \\
\text { VU: vulnerable; EN: endangered; CR: critically endangered. The species of least concern have no superscript. }\end{array}$} \\
\hline \multicolumn{9}{|c|}{$\begin{array}{l}\text { d The superscripts }+++,++ \text {, and }+ \text { indicate dominant species, subdominant species, and occasional species, } \\
\text { respectively. Rare species have no superscript. }\end{array}$} \\
\hline \multicolumn{9}{|c|}{$\begin{array}{l}\text { The partial correlation coefficient between bird number and DW. A positive value indicates that the bird number } \\
\text { increased with DW, whereas a negative value indicates a decrease. Values in bold indicate significant correlations at } \\
\text { the } p<0.05 \text { level. }\end{array}$} \\
\hline
\end{tabular}

The partial correlation coefficients showed that the birds' responses to the wind farm varied among species. Generally, however, the numbers of most dominant and subdominant birds were positively correlated with DW. In spring, $76.9 \%$ (10 of 13) of the dominant and subdominant bird species exhibited positive correlations, and 7 of them had significant positive correlations $(p<0.05)$. The percentage was $90.9 \%$ (10 of 11) in autumn, and 7 of the dominant and subdominant bird species had significant positive correlations $(p<0.05)$. Moreover, the total numbers of dominant birds and subdominant birds were all significantly and positively correlated with DW $(p<0.05)$ (Table 2$)$, indicating that dominant and subdominant birds tended to avoid the wind farm.

In contrast, the numbers of most rare birds were negatively correlated with DW. A total of $73.2 \%$ (52 of 71 ) and $60.3 \%$ (38 of 63) of the rare bird species exhibited negative correlations in spring and autumn, respectively. However, most of these correlations were nonsignificant because the rare birds were low in number and were recorded at only a few points. Nevertheless, the total number of rare birds exhibited a significant negative correlation $(p<0.05)$ with DW (Table 2), indicating that rare birds tended to approach the wind farm. The partial correlation coefficients of occasional birds, however, showed no obvious tendencies (Tables 1 and 2). Their responses to the wind farm were unclear and might be a transitional type between those of dominant/subdominant birds and rare birds.

The rare birds in the wetland comprised terrestrial birds and aquatic birds. These two groups had different responses to the wind farm. As shown in Table 1, 81.8\% (27 of 33) and 70.4\% (19 of 27) of the terrestrial bird species exhibited negative correlations with DW in spring and autumn, respectively. Moreover, the total number of terrestrial birds had a significant negative correlation with DW ( $p<0.05)$ (Table 2). By comparison, only $65.8 \%$ (25 of 38) and 52.8\% (19 of 36) of the aquatic birds were negatively correlated with DW in spring and autumn, respectively, and the correlation between the total number of terrestrial birds and DW was nonsignificant $(p>0.05)$. The above comparisons indicate that the aquatic birds were more alert to the wind farms, whereas the terrestrial birds better adapted to them. 
Table 2

Partial correlations between the total numbers of birds of different dominances and categories and the DW.

\begin{tabular}{|c|c|c|}
\hline \multirow[t]{2}{*}{ Dominance and category } & \multicolumn{2}{|c|}{ Partial correlation coefficient ${ }^{a}$} \\
\hline & Spring & Autumn \\
\hline Dominant & 0.543 & 0.700 \\
\hline Subdominant & 0.337 & 0.403 \\
\hline Occasional & -0.129 & 0.030 \\
\hline Rare & -0.428 & -0.343 \\
\hline Terrestrial $^{\mathrm{b}}$ & -0.208 & -0.212 \\
\hline Aquatic ${ }^{b}$ & -0.391 & -0.329 \\
\hline \multicolumn{3}{|c|}{ a Values in bold indicate significant correlations at the $p<0.05$ leve } \\
\hline
\end{tabular}

\section{Discussion}

\section{Bird community composition and the endangered species}

The dominant and subdominant bird species in the Rudong coastal wetland were all members of Charadriiformes. Most of the dominant and subdominant birds, such Calidris alpina, Calidris ruficollis, and Charadrius alexandrines, are common species in the West Pacific (IUCN, 2021). The dominant species recorded in this study were generally consistent with those detected in previous shorebird surveys of nearby wetlands (20 $50 \mathrm{~km}$ from our study area, without wind farms) in spring and autumn (Peng et al., 2017). However, previous surveys indicated that the dominant species accounted for $72.3 \sim$ $94.6 \%$ of the total number of aquatic birds (Peng et al., 2017), whereas the proportions were $~ 62 \%$ in this study. The proportions were much lower in our study area, probably because many dominant birds had been driven away by the wind farm.

Despite low bird number, the rare species comprised $~ 80 \%$ species in the entire community in the Rudong coastal wetland. In addition to aquatic birds, terrestrial birds (mostly Passeriformes) were also common among the rare species, accounting for $\sim 40 \%$ of them. Thus, we conclude that the wetland is rich in both aquatic and terrestrial bird species. Moreover, the proportion of rare birds ( 8\%) in our study (for comparison, only aquatic birds were considered) was much higher than that ( 3\%) in nearby wetlands (20 $180 \mathrm{~km}$ from our study area, without wind farms) (Ma et al., 2006; Ge et al., 2009; Peng et al., 2017), which indicates that rare birds were less affected by the wind farm than dominant birds.

Four endangered species, i.e., Calidris tenuirostris, Numenius madagascariensis, Platalea minor, and Calidris pygmaea were recorded during our survey. These 4 species breed in Eastern Siberia or Northeast China and winter in Southeast Asia to Australia. Recent reports showed that the estimated population sizes of Numenius madagascariensis, Platalea minor, and Calidris pygmaea in the EAAF were 32 thousand, 3500, and 450, respectively (Ma and Chen, 2018; IUCN, 2021). Calidris tenuirostris has a larger population consisting of $\sim 290$ thousand individuals (Ma and Chen, 2018). Despite the differences, the population sizes of all the species are decreasing, and habitat loss is the main reason for the decreases (IUCN, 2021). Thus, wind farm construction at their stopover sites might influence their future survival. In this study, Numenius madagascariensis, Platalea minor, and Calidris pygmaea were rarely observed in the Rudong coastal wetland, 
but Calidris tenuirostris was still a dominant species in the area. Correspondingly, their responses to the wind farm were different.

\section{Spatial responses of the bird community to the wind farm}

Our results showed that birds' responses to wind farms might vary depending on their dominance and category. Two tendencies were concluded from our results. First, the dominant and subdominant species ('dominant and subdominant' is written as 'dominant' below) tended to avoid the wind farm, whereas the rare species tended to approach them. Second, terrestrial birds were more adaptable than aquatic birds to the wind farm.

The variation in responses with dominance might be related to the following reasons. The dominant species, which are characterized by very high numbers of individuals, often fly in large groups, whereas the rare species fly singly or in small groups. Previous studies reported that larger bird groups have higher collision risks with obstacles because they have more social interactions (Croft et al., 2013; Croft et al., 2015), which can filter the information of obstacle cues and then disturb individuals' avoidance (Croft et al., 2013; Croft et al., 2015). Thus, the dominant species tended to avoid high collision risks, whereas the rare species could better avoid collisions when flying in the wind farm. Another reason for this phenomenon might be interspecific competition. Many studies have shown that dominant species usually govern the optimal resource, and subordinate species are often driven to seek novel resources to reduce competition (Pimm and Pimm, 1982; McKinney et al., 2011; Freshwater et al., 2014). In this study, the dominant species were more concentrated in the undisturbed portion of the wetland; thus, many rare species chose to forage in or near the wind farm, which were less utilized by the dominant species. In this respect, we think that wind farms might act as refuges for rare species.

The difference in responses between aquatic and terrestrial birds might be related to their morphologies. Aquatic birds, which mainly inhabit open seashores, usually have high wing aspect ratios, i.e., long and narrow wings (Norberg, 2004; Sheard et al., 2020). This wing form has a high lift-to-drag ratio and smaller wing - tip vortices, making it more suitable for gliding, soaring, and continuous flight (Norberg, 2004). However, long and narrow wings have low aerodynamic roll torque and a high moment of inertia, which reduces flight manoeuvrability and result in a higher turn radius and longer take-off distance (Norberg, 2004; McFarlane, 2014). Moreover, aquatic birds usually have short tails (Thomas and Balmford, 1995; Thomas, 1997), which is disadvantageous for maintaining stability and balance in flight and turning (Thomas and Balmford, 1995). In conclusion, aquatic birds have low flight manoeuvrability, which may hinder them from avoiding wind turbines and incline them to stay away from wind farms. In contrast, terrestrial birds inhabit cluttered environments such as forest and spend much of their foraging time climbing, clinging and hanging. They usually have low wing aspect ratios (i.e., broad and rounded wings) and long tails, which are more suitable for manoeuvrable short flights (Norberg, 2004). Correspondingly, terrestrial birds can better avoid obstacles such as wind turbines; thus, they are more adaptable to wind farms.

According to the above analysis, we conclude that the dominant aquatic birds were most negatively impacted by the wind farm. These birds include the endangered species Calidris tenuirostris and some vulnerable and near threatened species. In contrast, the rare terrestrial birds were least disturbed by even benefited from the wind farm in some respect. The situation was more complex for rare aquatic birds. Their group sizes result in low collision risk, and they have fewer dominant competitors in the wind farm. On the other hand, their morphology is disadvantageous for flying in wind farms. Thus, similar to those of the occasional species, their responses exhibited more uncertainty.

\section{Responses of the bird community to the $\mathbf{4}$ geographical factors}

The RDA results showed the approximate responses of the bird community to DW, DR, DS, and VA. Figure 2 indicates that the responses to DR, DS, and VA differed considerably between aquatic and terrestrial birds, but they did not vary significantly depending on dominance. For most aquatic birds, the numbers tended to increase with a decrease in DS and with increases in DR and VA, indicating that aquatic birds tended to occur in low tidal flats and bare lands and tended to avoid suburbs. The terrestrial birds, however, exhibited the reverse response; they were more likely to occur in the high 
marsh, vegetated areas, and areas near the suburbs. The above differences occurred because aquatic birds mainly feed on the macrobenthos, fishes, and aquatic plants (Collis et al., 2002; Wade and Hickey, 2008; Ma and Chen, 2018), which are mainly distributed in low bare flats and shallow water, whereas terrestrial birds mainly feed on Arthropoda and seeds, which are concentrated in the high marsh and vegetated areas (Muñoz et al., 2017). Moreover, the terrestrial birds in the wetland mainly came from the suburbs, whereas the aquatic birds were more unfamiliar with them, which induced their different responses to DR.

Fig.2 also shows that the biplot scores of DW are lower than those of DR, DS, and VA (i.e., the arrow length of DW is shorter than those of DR, DS, and VA), indicating that the contribution of DW to the spatial variation in the community is lower than those of the other 3 factors. Consequently, the bird responses to the wind farm inferred from partial correlation analysis are not obvious in Fig. 2 because the responses are covered by the effects of DR, DS, and VA. Based on the results of partial correlation analysis and RDA, we conclude that the bird community exhibits notable responses to the wind farm, but the responses are still inferior to those to suburbs, the sea and vegetation.

The stepwise regression equations described the variations of total bird numbers of different dominances and categories with the geographical factors (Table 3). The results indicate that the coefficient of DW was positive for dominant and subdominant birds and negative for rare birds; moreover, DW was eliminated from the equations of occasional and rare aquatic birds because the responses of these birds to wind farm were nonsignificant. The above results are consistent with those of partial correlation analysis. In addition, the coefficients of the other 3 factors are generally consistent with the RDA results. The occasional birds in autumn have no regression result, because their distribution exhibited great uncertainties and none of the factors could explain the variation of their number.

Table 3

Stepwise regression equations between the total numbers of birds of different dominances and categories and the geographical factors.

\begin{tabular}{|c|c|c|c|c|c|c|}
\hline \multirow{2}{*}{$\begin{array}{l}\text { Dominance and } \\
\text { category }\end{array}$} & \multicolumn{3}{|l|}{ Spring } & \multicolumn{3}{|l|}{ Autumn } \\
\hline & Equation a & $p$ & $R^{2}$ & Equation a & $p$ & $R^{2}$ \\
\hline Dominant & $\begin{array}{l}N=0.070 D W+0.209 D R- \\
0.017 V A+297\end{array}$ & $\begin{array}{l}< \\
0.001\end{array}$ & 0.646 & $\begin{array}{l}N=0.054 D W+0.098 D R- \\
0.043 D S-0.011 V A+308\end{array}$ & $\begin{array}{l}< \\
0.001\end{array}$ & 0.757 \\
\hline Subdominant & $\begin{array}{l}N=0.020 \mathrm{DW}+0.099 \mathrm{DR}- \\
0.009 \mathrm{VA}+133\end{array}$ & $\dot{0} 001$ & 0.609 & $\begin{array}{l}N=0.019 D W+0.059 D R- \\
0.006 V A+93\end{array}$ & $<.001$ & 0.536 \\
\hline Occasional & $\begin{array}{l}N=0.028 \mathrm{DR}-0.010 \mathrm{DS}- \\
0.002 \mathrm{VA}+39\end{array}$ & $<.001$ & 0.546 & - & - & - \\
\hline Rare & $\begin{array}{l}N=-0.008 D W+0.013 D R \\
-0.001 V A+67\end{array}$ & 0.001 & 0.311 & $N=-0.005 \mathrm{DW}-0.002 \mathrm{VA}+71$ & 0.001 & 0.299 \\
\hline Terrestrial $^{\mathrm{b}}$ & $\begin{array}{l}N=-0.004 \mathrm{DW}-0.013 \mathrm{DR} \\
+0.011 \mathrm{DS}+26\end{array}$ & $<.001$ & 0.505 & $\begin{array}{l}N=-0.002 \mathrm{DW}-0.007 \mathrm{DR}+ \\
0.006 \mathrm{DS}+15\end{array}$ & $<.001$ & 0.522 \\
\hline Aquatic $^{b}$ & $\begin{array}{l}N=0.025 \mathrm{DR}-0.014 \mathrm{DS}- \\
0.001 \mathrm{VA}+44\end{array}$ & $<.001$ & 0.447 & $\begin{array}{l}N=0.013 \mathrm{DR}-0.011 \mathrm{DS}- \\
0.002 \mathrm{VA}+52\end{array}$ & 0.001 & 0.291 \\
\hline \multicolumn{7}{|c|}{$\begin{array}{l}\text { a } \text { : total bird number; DW: distance to the wind farm boundary; DR: distance to the suburbs; DS: distance to the sea; } \\
\text { VA: vegetation area surrounding each census point. }\end{array}$} \\
\hline
\end{tabular}

\section{Conclusion}


As an important stopover site of birds in the EAAF, the Rudong coastal wetland comprises rich bird species.

Charadriiformes accounts for an extremely high proportion of the total bird number, and Charadriiformes and Passeriformes comprise the majority of the species in the community.

Numerous studies have demonstrated negative effects of wind farms on birds, which indicates a conflict between bird conservation and wind power development in coastal areas of East China. Nevertheless, our study suggested that birds' responses to wind farms might vary according to their dominance and category. The most negatively impacted birds were the dominant aquatic birds in the wetlands. These birds included the endangered species Calidris tenuirostris, the vulnerable species Chroicocephalus saundersi, and some near threatened species. Wind farms might compress the habitats of these species and then contribute to future population declines. Thus, the protection degrees of these species in the area should be upgraded. We think that retaining sufficient undisturbed intertidal mudflats, which are major foraging places for these birds, will be crucial for maintaining their population sizes in the future. The occasional birds and rare aquatic birds in the area, including the critically endangered species Calidris pygmaea, the endangered species Numenius madagascariensis and Platalea minor, and various vulnerable and near threatened species, exhibit great uncertainties in their responses to wind farms. They might be disturbed by wind farms but not significantly so. We think that monitoring their population dynamics in the area is a major task for the short term. Finally, the rare terrestrial birds in the area might accept the wind farms as refuges under interspecific competition, and they can better adapt to the wind farm environment. Thus, we conclude that these species, including the vulnerable species Acrocephalus tangorum and Locustella pleskei, would be least disturbed by or would even benefit somewhat from future wind farm construction.

Wind power development has altered the wetland environment and bird habitat selection in the Rudong coastal wetland. Our two-year observation of the area will provide a guide for future bird conservation in wetlands with wind farms.

\section{Declarations}

Availability of Data and Material The Landsat $8 \mathrm{OLI}$ image used here are available at http://eds.ceode.ac.cn/nuds/freedataquery.

Acknowledgements We sincerely thank the editors and the anonymous reviewers for their valuable comments and suggestions for this manuscript. We also thank Shanshan Chang, Lijuan Chen, Chuangqi Hu, and Yuqiao Hou for their help in our bird survey. We greatly appreciate Nanjing Normal University to support this work.

Author Contributions YC contributed to the study conception and design. Data acquisition was performed by $Y C, Y Z, G W$, DD and data analysis were performed by WZ and CT. The original draft was written by $Y C$ and $Y Z$ reviewed and edited subsequent versions of the manuscript.

Funding This work was supported by the National Natural Science Foundation of China (No. 41671428) and Nanjing Normal University.

Data Availability All data are presented in the paper

Code Availability The R-code generated and analyzed is available upon request from the corresponding author.

Ethics approval This paper contains findings of our original research. The Law of the People's Republic of China on the Protection of Wildlife and IUCN Policy Statement on Research Involving Species at Risk of Extinction were followed during the study. No animal was harmed or captured. No animal or plant samples were taken from the study area. We adhered to the ethical standards in this study and in production of this manuscript.

Consent to Participate Not applicable

Page 15/19 
Consent for Publication The article submitted herewith contains the findings of our original research, is not under consideration for publication elsewhere, and is approved by all authors of this manuscript.

Conflicts of interest/Competing Interests Authors declare no conflict of interest

\section{References}

1. Bai QQ, Chen JZ, Chen ZH, Dong GT, Dong JT, Dong WX, Fu VWK, Han YX, Lu G, Li J, Liu Y, Lin Z, Meng DR, Martinez J, Ni GH, Shan K, Sun RJ, Tian SX, Wang FQ, Xu ZW, Yu Y, Yang J, Yang ZD, Zhang L, Zhang M, Zeng XW (2015) Identification of coastal wetlands of international importance for waterbirds: a review of China Coastal Waterbird Surveys 2005-2013. Avian Research 6:12

2. Bibby CJ, Burgess ND, Hill DA, Mustoe SH (2000) Bird census techniques (Second edition). Academic Press, London

3. Borcard D, Gillet F, Legendre P (2011) Numerical ecology with R. Springer, New York

4. Cao L, Tang S, Wang X, Barter M (2009) The importance of eastern China for shorebirds during the non-breeding season. Emu-Austral Ornithology 109:170-178

5. Chapman KA, Reich PB (2007) Land use and habitat gradients determine bird community diversity and abundance in suburban, rural and reserve landscapes of Minnesota, USA. Biol Cons 135:527-541

6. Collis K, Roby DD, Craig DP, Adamany S, Adkins JY, Lyons DE (2002) Colony size and diet composition of piscivorous waterbirds on the lower Columbia River: implications for losses of Juvenile Salmonids to avian predation. Trans Am Fish Soc 131:537-550

7. Croft S, Budgey R, Pitchford JW, Wood AJ (2013) The influence of group size and social interactions on collision risk with obstacles. Ecological Complexity 16:77-82

8. Croft S, Budgey R, Pitchford JW, Wood AJ (2015) Obstacle avoidance in social groups: new insights from asynchronous models. Journal of the Royal Society Interface 12:20150178

9. Freshwater C, Ghalambor CK, Martin PR (2014) Repeated patterns of trait divergence between closely related dominant and subordinate bird species. Ecology 95(8):2334-2345

10. Ge ZM, Zhou X, Wang TH, Wang KY, Pei E, Yuan X (2009) Effects of vegetative cover changes on the carrying capacity of migratory shorebirds in a newly formed wetland, Yangtze River Estuary, China. Zoological Studies 48(6):769-779

11. Grodsky SM, Jennelle CS, Drake D (2013) Bird mortality at a wind-energy facility near a wetland of international importance. The Condor 115(4):700-711

12. He ZX, Xu SC, Shen WX, Zhang H, Long RY, Yang H, Chen H (2016) Review of factors affecting China's offshore wind power industry. Renew Sustain Energy Rev 56:1372-1386

13. Herbert GMJ, Iniyan S, Amutha D (2014) A review of technical issues on the development of wind farms. Renew Sustain Energy Rev 32:619-641

14. Hilgerloh G, Michalik A, Raddatz B (2011) Autumn migration of soaring birds through the Gebel El Zeit Important Bird Area (IBA), Egypt, threatened by wind farm projects. Bird Conservation International 21(4):365-375

15. IUCN (2021) The IUCN red list of threatened species. Version 2021-1. https://www.iucnredlist.org/. Accessed 12 February 2021

16. Jackson MV, Fuller RA, Gan XJ, Li J, Mao DH, Melville DS, Murray NJ, Wang ZM, Choi CY (2021) Dual threat of tidal flat loss and invasive Spartina alterniflora endanger important shorebird habitat in coastal mainland China. J Environ Manage 278:111549

17. Kumar Y, Ringenberg J, Depuru SS, Devabhaktuni VK, Lee JW, Nikolaidis E, Andersen B, Afjeh A (2016) Wind energy: trends and enabling technologies. Renew Sustain Energy Rev 53:209-224

18. Lenz M (1990) The breeding bird communities of three Canberra suburbs. Emu-Austral Ornithology 90:145-153

Page 16/19 
19. Li JG, Yang WH, Li Q, Pu LJ, Xu Y, Zhang ZQ, Liu LL (2018) Effect of reclamation on soil organic carbon pools in coastal areas of eastern China. Frontiers of Earth Science 12(2):339-348

20. Lin QY, Yu S (2018) Losses of natural coastal wetlands by land conversion and ecological degradation in the urbanizing Chinese coast. Sci Rep 8:15046

21. Ma ZJ, Chen SH (2018) The birds in the sea and wetlands of China. Hunan Science and Technology Press, Changsha (in Chinese)

22. Ma ZJ, Choi CY, Gan XJ, Zheng S, Chen JK (2006) The importance of Jiuduansha Wetlands for shorebirds during northward migration: energy-replenishing sites or temporary stages? Stilt 50:54-57

23. Ma ZJ, Wang Y, Gan XJ, Li B, Cai YT, Chen JK (2009) Waterbird population changes in the wetlands at Chongming Dongtan in the Yangtze River Estuary, China. Environ Manage 43:1187-1200

24. Marques AT, Santos CD, Hanssen F, Muñoz A, Onrubia A, Wikelski M, Moreira F, Palmeirim JM, Silva JP (2020) Wind turbines cause functional habitat loss for migratory soaring birds. J Anim Ecol 89:93-103

25. McFarlane LA (2014) Avian wing morphology: intra- and inter- specific effects on take-off performance and muscle function in controlling wing shape over the course of the wing stroke. Dissertation, The University of Leeds

26. McKinney RA, Raposa KB, Cournoyer RM (2011) Wetlands as habitat in urbanizing landscapes: Patterns of bird abundance and occupancy. Landscape Urban Planning 100:144-152

27. Muñoz CE, Ippi S, Celis-Diez JL, Salinas D, Armesto JJ (2017) Arthropods in the diet of the bird assemblage from a forested rural landscape in Northern Chiloé Island, Chile: a quantitative study. Ornitologia Neotropical 28:191-199

28. Newton I, Little B (2009) Assessment of wind-farm and other bird casualties from carcasses found on a Northumbrian beach over an 11-year period. Bird Study 56:158-167

29. Niemuth ND, Estey ME, Reynolds RE, Loesch CR, Meeks WA (2006) Use of wetlands by spring-migrant shorebirds in agricultural landscapes of North Dakota's drift prairie. Wetlands 26(1):30-39

30. Norberg UML (2004) Bird flight. Acta Zool Sin 50(6):921-935

31. Peng HB, Anderson GQA, Chang Q, Choi CY, Chowdhury SU, Clark NA, Gan XJ, Hearn RD, Li J, Lappo EG, Liu WL, Ma ZJ, Melville DS, Phillips JF, Syroechkovskiy EE, Tong MX, Wang SL, Zhang L, Zöckler C (2017) The intertidal wetlands of southern Jiangsu Province, China - globally important for spoon-billed sandpipers and other threatened waterbirds, bur facing multiple serious threats. Bird Conservation International 27(3):305-322

32. Pimm SL, Pimm JW (1982) Resource use, competition, and resource availability in Hawaiian honeycreepers. Ecology 63(5):1468-1480

33. Plonczkier P, Simms IC (2012) Radar monitoring of migrating pink-footed geese: behavioural responses to offshore wind farm development. J Appl Ecol 49:1187-1194

34. Pruett CL, Patten MA, Wolfe DH (2009) It's not easy being green: wind energy and a declining grassland bird. Bioscience 59(3):257-262

35. Ralph CJ, Droege S, Sauer JR (1995) Managing and monitoring birds using point counts: standards and applications. Page 161-168 in Ralph CJ, Sauer JR, Droege S. editors Monitoring bird populations by point counts. USDA Forest Service, General Technical Report PSW-GTR-149

36. Sheard C, Neate-Clegg MHC, Alioravainen N, Jones SEl, Vincent C, MacGregor HEA, Bregman TP, Claramunt S, Tobias JA (2020) Ecological drivers of global gradients in avian dispersal inferred from wing morphology. Nat Commun $11: 2463$

37. Thomas ALR (1997) On the tails of birds. Bioscience 47(4):215-225

38. Thomas ALR, Balmford A (1995) How natural selection shapes birds' tails. Am Nat 146(6):848-868

39. Villegas-Patraca R, Cabrera-Cruz SA, Herrera-Alsina L (2014) Soaring migratory birds avoid wind farm in the Isthmus of Tehuantepec, Southern Mexico. PLoS One 9(3):e92462 
40. Wade S, Hickey R (2008) Mapping migratory wading bird feeding habitats using satellite imagery and field data, Eighty-Mile Beach, Western Australia. J Coastal Res 24(3):759-770

41. Wang JJ, Zou XQ, Yu WW, Zhang DJ, Wang T (2019) Effects of established offshore wind farms on energy flow of coastal ecosystems: A case study of the Rudong offshore wind farms in China. Ocean Coast Manag 171:111-118

42. WWEA (2021) Worldwide wind capacity reaches 744 gigawatts - An unprecedented 93 gigawatts added in 2020. https://wwindea.org/worldwide-wind-capacity-reaches-744-gigawatts/. Accessed 5 April 2021

43. Yang JB, Liu QY, Li X, Cui XD (2017) Overview of wind power in China: status and future. Sustainability 9:1454

44. Yang ZY, Lagassé BJ, Xiao H, Jackson MV, Chiang CY, Melville DS, Leung KSK, Li J, Zhang L, Peng HB, Gan XJ, Liu WL, Ma ZJ, Choi CY (2020) The southern Jiangsu coast is a critical moulting site for Spoon-billed Sandpiper Calidris pygmaea and Nordmann's Greenshank Tringa guttifer. Bird Conservation International 30(4):649-660

45. Yong DL, Jain A, Liu Y, Iqbal M, Choi CY, Crockford NJ, Millingtong S, Provencher J (2018) Challenges and opportunities for transboundary conservation of migratory bird in the East Asian-Australasian flyway. Conserv Biol 32(3):740-743

46. Yong DL, Liu Y, Low BW, Española CP, Choi CY, Kawakami K (2015) Migratory songbirds in the East Asian-Australasian Flyway: a review from a conservation perspective. Bird Conservation International 25(1):1-37

\section{Figures}
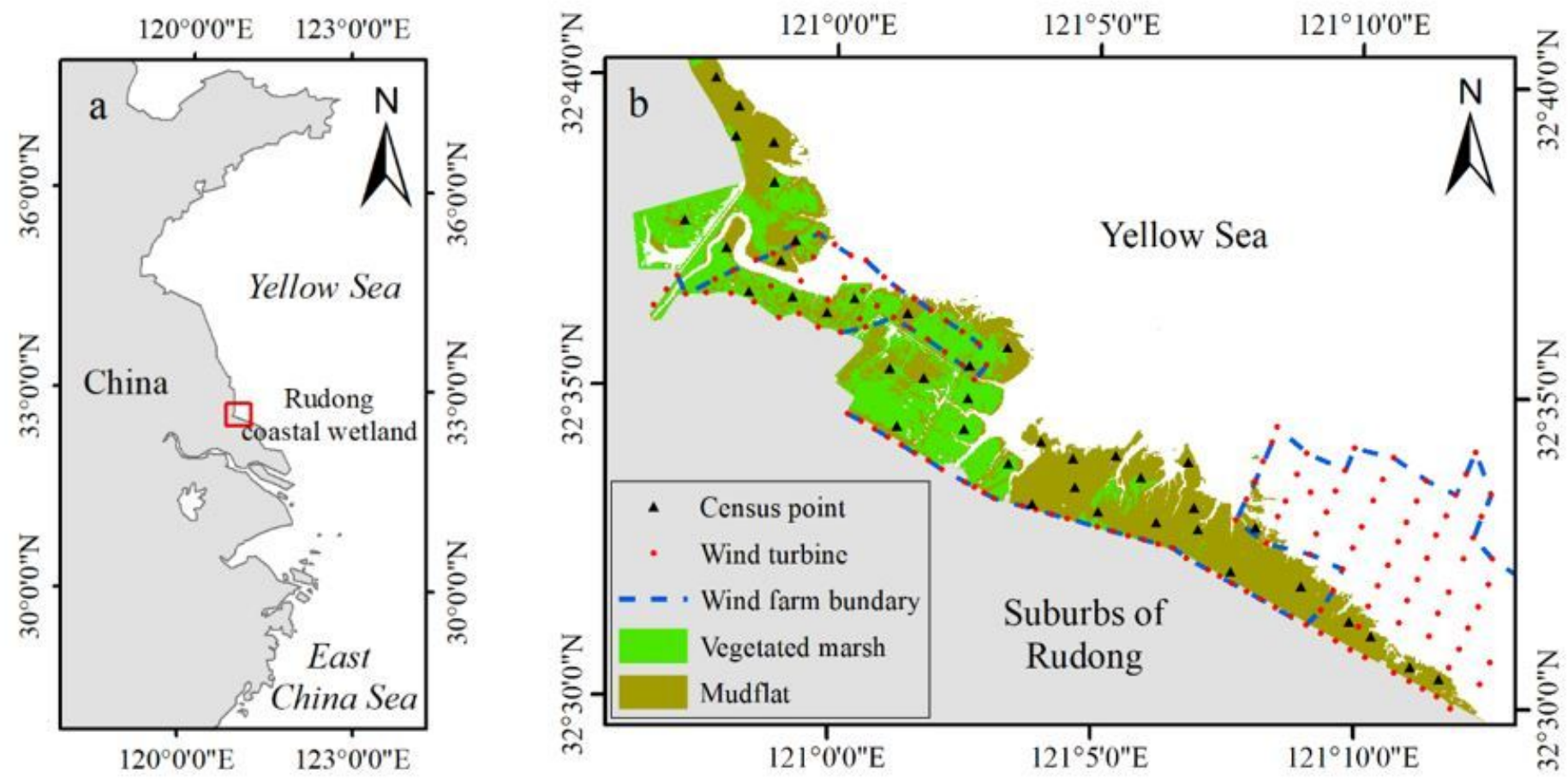

\section{Figure 1}

(a) Location of the study area and (b) distribution of census points and wind turbines in the Rudong coastal wetland. Note: The designations employed and the presentation of the material on this map do not imply the expression of any opinion whatsoever on the part of Research Square concerning the legal status of any country, territory, city or area or of its authorities, or concerning the delimitation of its frontiers or boundaries. This map has been provided by the authors. 

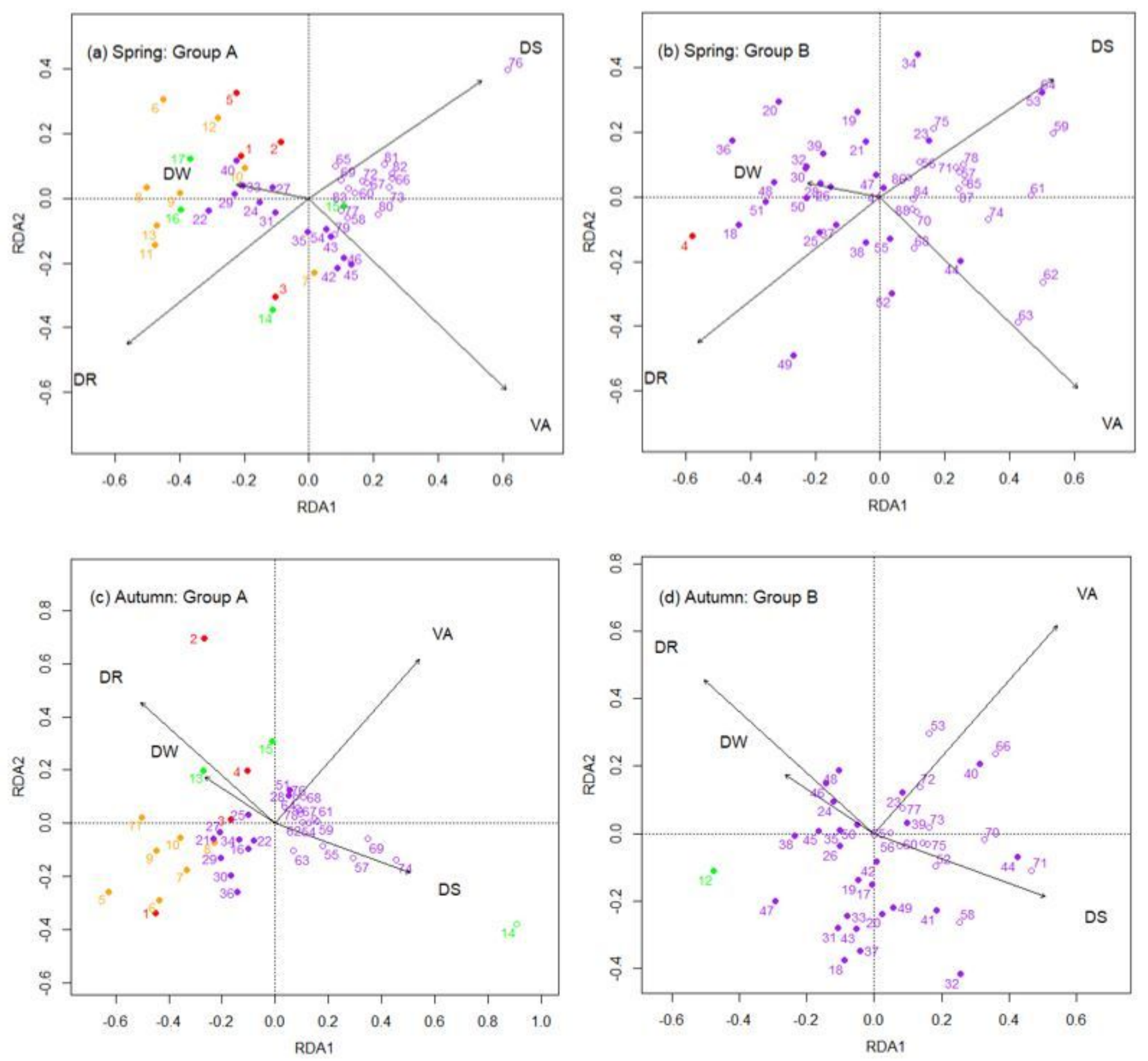

Figure 2

Results of redundancy analysis of bird numbers and geographical factors. (a) Spring: Group A; (b) Spring: Group B; (c) Autumn: Group A; (d) Autumn: Group B. The species scores in Group A have been magnified by 2.5 times, and those in Group B have been magnified by 15 times. The colours of the circles indicate the dominance of the species: red, orange, green, and purple represent the dominant, subdominant, occasional, and rare species, respectively. The solid circles indicate aquatic birds, whereas the open circles indicate terrestrial birds. Numbers beside the circles are the species numbers in Table 1. DW: distance to the wind farm boundary; DR: distance to the suburb; DS: distance to the sea; VA: vegetation area surrounding each census point. 